Article

\title{
Influence of Model Simplifications Excitation Force in Surge for a Floating Foundation for Offshore Wind Turbines
}

\author{
Morten Thøtt Andersen ${ }^{1, *}$, Dennis Hindhede ${ }^{2, \dagger}$ and Jimmy Lauridsen ${ }^{2, \uparrow}$ \\ 1 Department of Civil Engineering, Aalborg University, Sofiendalsvej 11, 9200 Aalborg SV, Denmark \\ 2 Department of Civil Engineering, Aarhus University, Inge Lehmanns Gade 10, 8000 Aarhus C, \\ Denmark; E-Mails: dhi@ramboll.com (D.H.); lauridsen1988@gmail.com (J.L.) \\ $\dagger$ These authors contributed equally to this work. \\ * Author to whom correspondence should be addressed; E-Mail: mta@civil.aau.dk; \\ Tel.: +45-9940-8545; Fax: +45-9940-8552.
}

Academic Editor: John Ringwood

Received: 10 December 2014 / Accepted: 14 April 2015 / Published: 22 April 2015

\begin{abstract}
As offshore wind turbines move towards deeper and more distant sites, the concept of floating foundations is a potential technically and economically attractive alternative to the traditional fixed foundations. Unlike the well-studied monopile, the geometry of a floating foundation is complex and, thereby, increases the difficulty in wave force determination due to limitations of the commonly used simplified methods. This paper deals with a physical model test of the hydrodynamic excitation force in surge on a fixed three-columned structure intended as a floating foundation for offshore wind turbines. The experiments were conducted in a wave basin at Aalborg University. The test results are compared with a Boundary Element Method code based on linear diffraction theory for different wave force regimes defined by the column diameter, wave heights and lengths. Furthermore, the study investigates the influence of incident wave direction and stabilizing heave-plates. The structure can be divided into primary, secondary and tertiary parts, defined by the columns, heave-plates and braces to determine the excitation force in surge. The test results are in good agreement with the numerical computation for the primary parts only, which leads to simplified determination of peak frequencies and corresponding dominant force regime.
\end{abstract}

Keywords: wave force; surge; experimental; semi-submersible; offshore; floating foundation 


\section{Introduction}

One of the most well-known and currently preferred renewable energy sources is wind power. The wind power resource is on average 50\% higher offshore than onshore; however, the cost to install and run offshore wind power units is around double [1]. This has raised the challenge of optimizing the economical perspectives of offshore wind power. The generation of wind power is mainly located in areas within a distance of 5-50 km from the coastline, where the water depth is generally greater than $20 \mathrm{~m}$. Studies have shown that the traditional fixed foundations will not be economically viable for offshore wind turbines in waters deeper than $30 \mathrm{~m}$ [2], which is why the concept of a floating foundation is a promising alternative. Four main concept types have been developed: semi-submersible, TLP, spar buoy and barge [3]. This paper will focus on the conceptual semi-submersible prototype, see Figure 1.
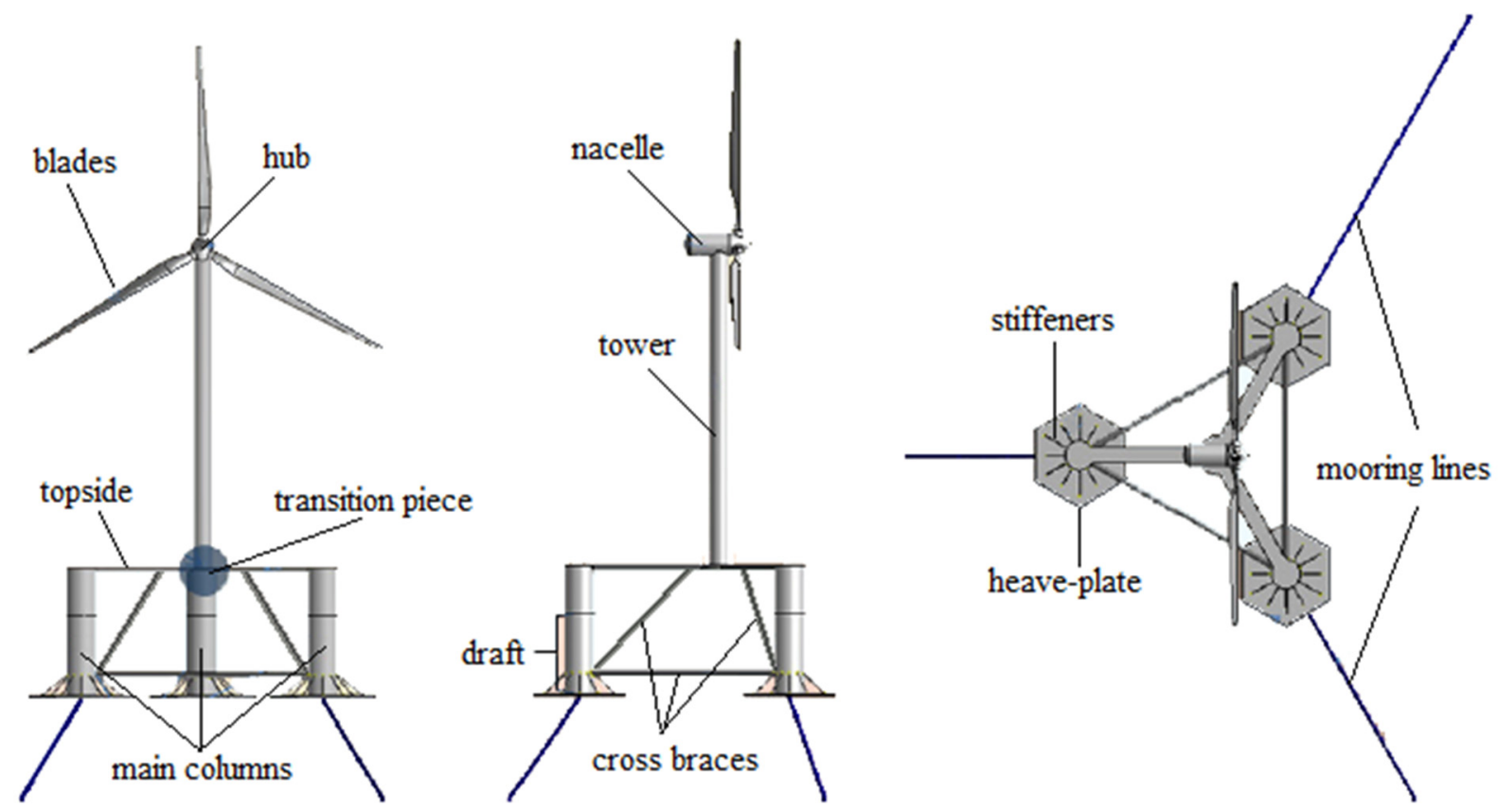

Figure 1. Conceptual semi-submersible prototype.

As conceptual designs of floating foundations are proposed, the evaluation of structural response is of high priority and a key element in the design [4]. This requires a fundamental determination and physical understanding of the wave force acting on the structure. One of the important parameters due to its high influence on hydrodynamic impact is the geometry. The dominating wave force regimes on cylindrical structures depend on the ratios of wave height to column diameter (H/D) and of column diameter to wavelength (D/L) [5]. For many reasons like separation, reflection, omni-directionality, three-dimensionality, turbulence, diffraction, refraction, flow-induced vibrations, etc., a full structural design is beyond current computational capacity [6], so the current design philosophy for wave induced loads is often determined by empirical or first principle calculations of simple models and geometry (such as Morison [7]), which is why the validity of the applied methods for more complex structures is questionable [8].

In a recent test campaign on a semi-submersible $1 / 50$ scale model in a wave basin, the recommendation was more research focusing on validation of the hydrodynamic loads by component-level tests [8]. Further, the modelling of wave loads by a definition of the wave spectrum is 
seen as a sufficient approach to a given design. A frequency domain analysis shows that specific wave periods of regular waves and the direction of incident waves have significant effects on the excitation forces and motion of the structure [9]. The analysis of the global motion response for a semi-submersible floating wind turbine has previously been examined [10]. The study concluded that an uncoupled frequency domain analysis was sufficient to assess the global motion response as long as the aerodynamic forces were taken into account. For numerical evaluation of wave/structure interaction, the Boundary Element Method (BEM) is used to compute diffraction/radiation theory in related studies of wave force on a semi-submerged platform [11].

This paper deals with experimental analysis of the wave excitation force in surge on a conceptual prototype model of a semi-submersible foundation for an offshore wind turbine from Ramboll [12]. The concept is considered in three structural parts: columns (primary), heave-plates (secondary) and braces (tertiary). Because of the complex geometry, it is appropriate to analyse the excitation of wave induced loads on the structure in a component level test by analysing the influence of different parameters. The wave basin model test (Froude-based scale; 1/80) is conducted to observe and examine the wave load in surge. The model was fixed to simplify the influence of dynamic parameters by neglecting the motions.

The wave induced load is measured and compared with computed results of the diffraction/radiation problem in the open source BEM code, Nemoh [13], in order to obtain a feasible difference of simplified calculations of a complex geometric structure. Nemoh is based on linear diffraction theory, which neglects viscous effect. The diffraction effects are expected to be dominating due to the ratios H/D $(0.01<\mathrm{H} / \mathrm{D}<1.5)$ and $\mathrm{D} / \mathrm{L}(\pi \mathrm{D} / \mathrm{L}>0.5)$, which make Nemoh applicable for the numerical computation. The model scaled experiment will together with the computed Nemoh results provide the basis of a parametrical analysis of the influence of a complex geometry on the excitation force in surge on a floating foundation prototype. Table 1 lists the parameter variation ranges.

Table 1. Parameters of tested wave set.

\begin{tabular}{cccc}
\hline Parameter & Range & Ratio & Range \\
\hline Wave height $(\mathrm{H})$ & $2 \mathrm{~cm}-16 \mathrm{~cm}$ & $\pi \mathrm{D} / \mathrm{L}$ & $0.05-1.26$ \\
Wave period $(\mathrm{T})$ & $0.4 \mathrm{~s}-2.5 \mathrm{~s}$ & (diffraction parameter) & \\
Wave length $(\mathrm{L})$ & $0.25 \mathrm{~m}-5.87 \mathrm{~m}$ & H/D & $0.20-1.60$ \\
Incident wave direction $(\theta)$ & $0^{\circ}-90^{\circ}$ & (wave steepness) & \\
\hline
\end{tabular}

\section{Method}

\subsection{Background}

The comparison between measurements and Nemoh computation is, among other things, assumptions based on the validity of wave theories and dominant wave force regime. The experiments are a combination of variations in the parameters' wave frequency ( $\mathrm{f}=1 / \mathrm{T}), \theta, \mathrm{H}$, and the geometrical modification by removing heave-plates and replacing them with cylinder plugs.

The variables $\mathrm{H}$ and $\mathrm{f}$ indicate the validity of wave theory in constant water depth (d) [8], where $\mathrm{H}$, $\mathrm{L}$ and $\mathrm{D}$ determine the dominant force regimes [5]. The variation of $\mathrm{f}$ with constant $\mathrm{H}$ leads to an analysis of frequency dependable forces, whereas the variation of $\mathrm{H}$ with constant $\mathrm{f}$ leads to an analysis of contributions of viscous effects and omission of linear wave theory. Because the geometry of the 
different structural parts affects the dominant force regime, it is appropriate to divide the structure into primary, secondary and tertiary parts based on their dominance, since the areas of wet surface (parts below Still Water Level (SWL)) and submerged volume are considered. In both cases, the columns are dominating since they are considered the primary structural part (see Figure 2a and Table 2). Figure $2 \mathrm{~b}$ shows that by f-variation (scattered plot) the columns are within the inertia and diffraction area, where $\mathrm{H}$-variation determines the significance of drag effects in dominant wave force regime. The ratio between the total frontal wet areas is $A_{\left(\theta=0^{\circ}\right)} / A_{\left(\theta=30^{\circ}\right)}=1.4$, which might lead to potential shadow effects due to in-line positioning of columns in incident wave direction.

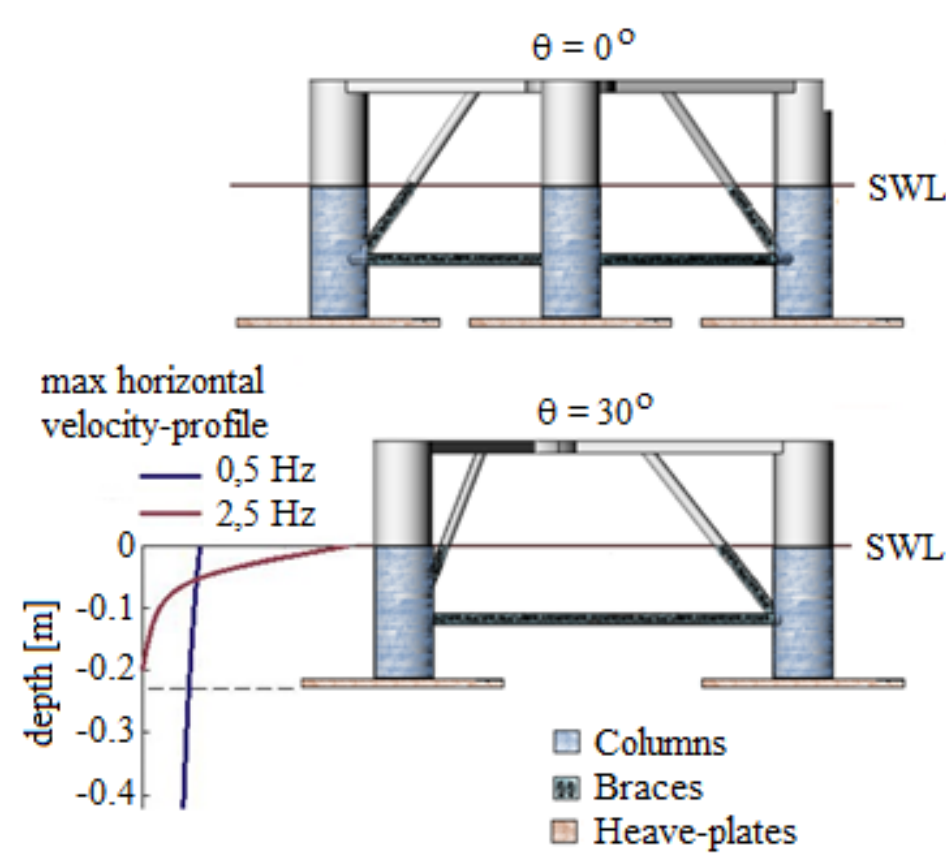

(a)

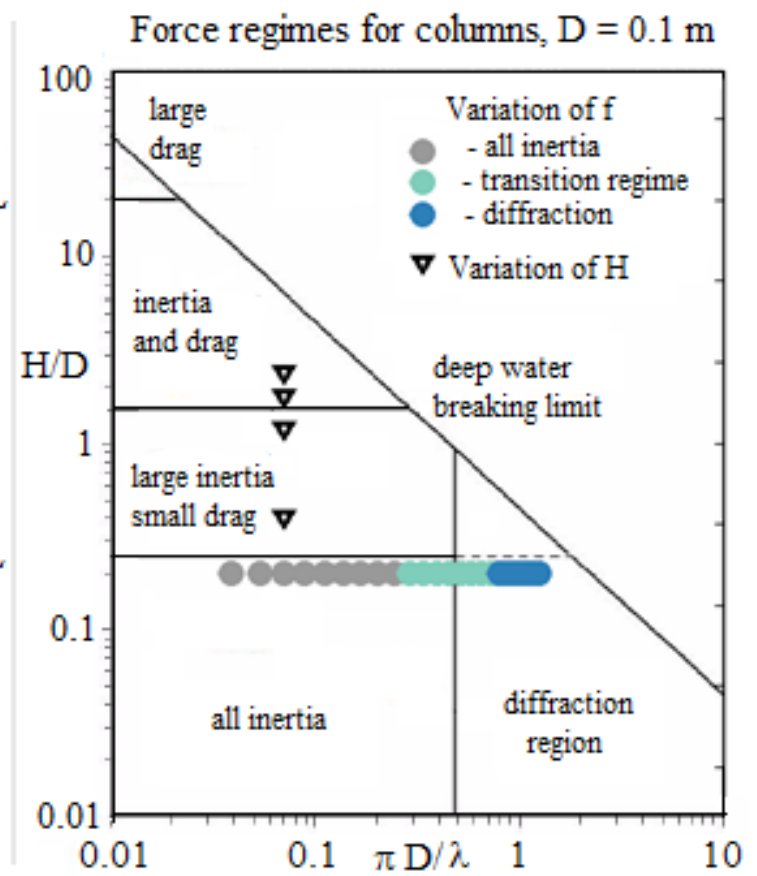

(b)

Figure 2. (a) Sub-divided frontal wet areas of model in incident wave direction, with illustration of velocity profile (b) Different wave force regimes of tested waves [5].

For low frequency waves, the maximum velocity profile is almost constant down the structure (see Figure 2a), which is why all structural parts are considered to be affected equally. For high frequency waves, the heave-plates have no influence because of the unaffected particles in depth. The viscous effects depend on the particle velocity and will primarily occur around the braces because of the small diameter and above the heave-plates because of the large horizontal area.

Table 2. Frontal wet areas (in incident wave direction), volume and force regime of wetted surface.

\begin{tabular}{|c|c|c|c|c|c|}
\hline \multirow{2}{*}{ Structural part } & \multicolumn{2}{|c|}{ Area distribution } & \multirow{2}{*}{ Volume } & \multicolumn{2}{|c|}{ Dominant effect } \\
\hline & $\theta=0^{\circ}$ & $\theta=\mathbf{3 0} 0^{\circ}$ & & Low freq. & High freq. \\
\hline Columns & $74 \%$ & $70 \%$ & $69 \%$ & Inertia & Diffraction \\
\hline Heave-plates & $7 \%$ & $6 \%$ & $21 \%$ & Small drag & No influence \\
\hline Braces & $19 \%$ & $24 \%$ & $10 \%$ & Inertia & Small drag \\
\hline
\end{tabular}




\subsection{Loads on Large Cylinders}

The forces of a flow acting on a body can be calculated as the pressure and shear forces integrated over the instantaneous wetted area. The solution can be found by solving the Navier-Stokes equations, which are a nonlinear set of differential equations. Today, there is no closed-form solution for the non-simplified equations, and the solution is commonly found by the use of Computational Fluid Dynamics (CFD). The major disadvantage of using CFD is the high demand of processing power and calculation time, since it is normal in hydrodynamics to make the simplifications, assuming incompressible, inviscid and irrotational flow. The potential theory follows these assumptions, and it is widely used in fluid mechanics despite its limitations [14].

When D/L becomes large, the columns will generate both reflected and diffracted waves which affect the incident wave. This process is, in general, termed diffraction and is important when the ratio $\mathrm{D} / \mathrm{L}>0.2$, because it cannot be assumed anymore that the wave is not modified by the interactions with the body [8]. The total velocity potential $(\varphi)$ can be seen as the linear sum (1) of the incident wave potential $\left(\varphi_{w}\right)$, the scattered/diffracted wave potential $\left(\varphi_{S}\right)$ and radiated wave potential $\left(\varphi_{R}\right)$, which is zero due to fixation of model. The total velocity potential has to satisfy the Laplace equation (2) within the fluid domain, subjected to appropriate boundary conditions. McCamy \& Fuchs [15] obtained the solution for diffraction force exerted on a circular cylinder and it is applicable as an analytical reference for lower and upper limits in terms of excitation force on one and three columns respectively:

$$
\begin{gathered}
\varphi=\varphi_{w}+\varphi_{s}+\varphi_{R} \\
\nabla^{2} \varphi=0
\end{gathered}
$$

Nemoh computes the linear partial differential equations of velocity potential by a boundary integral method and solves the Laplace Equation (2), where the 3-dimensional problem is described by a surface problem (panel method). For the computed results, a full model mesh (1,590 panels) and a mesh with primary structural parts only (three columns: 264 panels) are applied so as to observe differences, see Figure 3. A convergence analysis of panel size shows a divergence below $1 \%$.
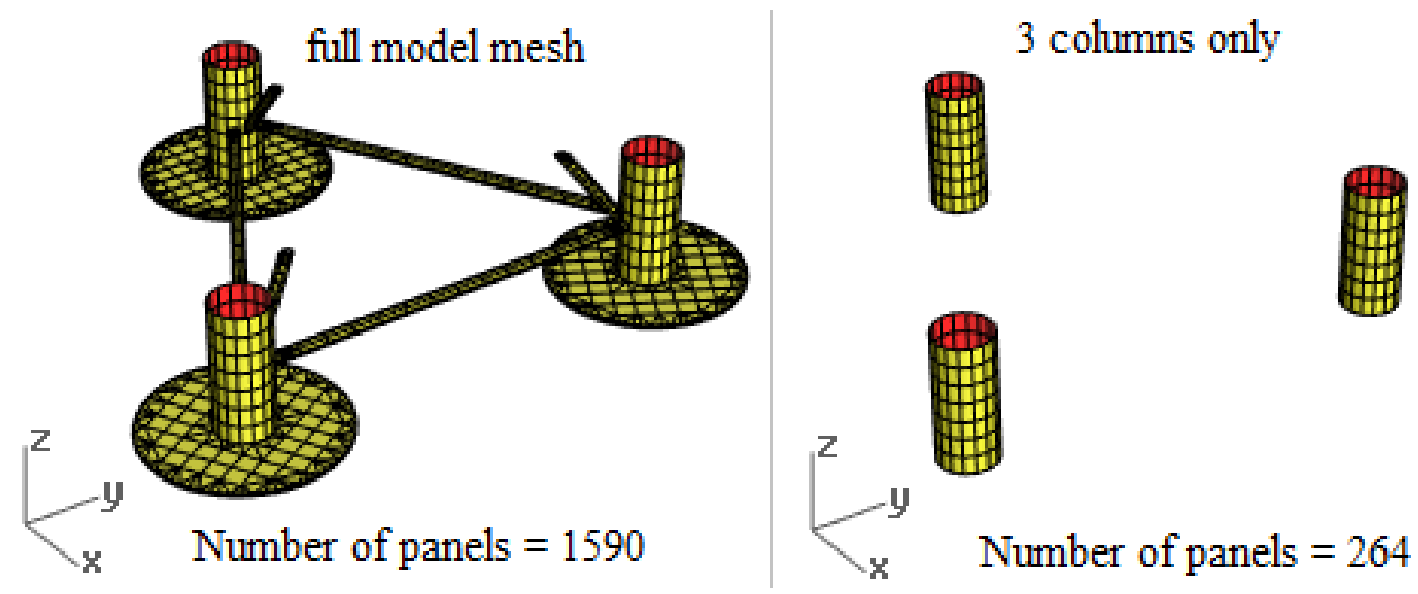

Figure 3. Meshes of the wetted structure applied in Nemoh (ratio in number of panels: $\sim 6$ ). 


\section{Experimental Setup}

The experiments were conducted in a wave basin at The Hydraulic and Coastal Engineering Laboratory, Aalborg University, Denmark between 11/8-2014 and 26/9-2014. The software Awasys 6 [16] controls the wave maker by inputs of $\mathrm{f}$ and $\mathrm{H}$. Measurements of $\mathrm{H}$ by wave gauges are used in data processing. The tests were conducted with a water depth of $0.65 \mathrm{~m}$ and a temperature of $\sim 20{ }^{\circ} \mathrm{C}$. Figure 4 illustrates the test setup. The wave maker is at one end of the basin with an absorbing gravel beach installed at the opposite end.

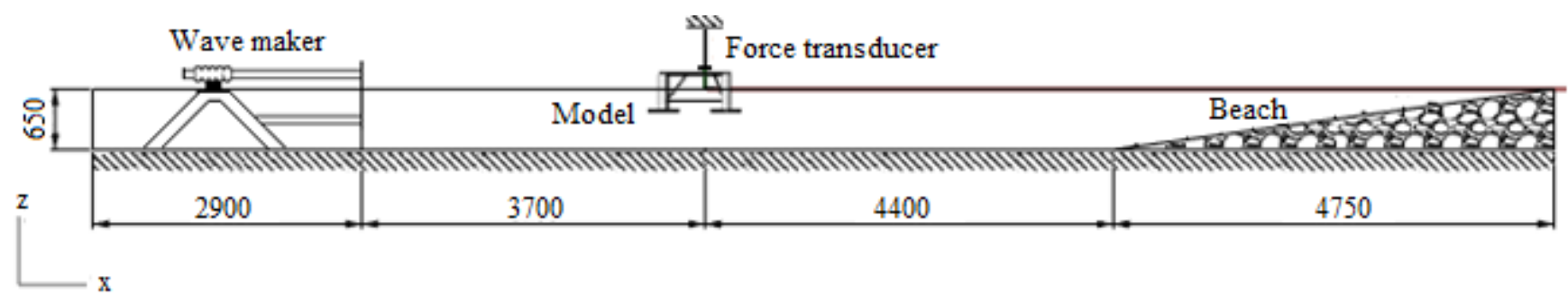

Figure 4. Illustration of experimental setup of wave basin [mm].

The paddle system is of the snake front type with translator movements. A Multi-Axis Force/Torque (FT) Sensor (ATI F/T: Delta IP65, 330-30: http://www.ati-ia.com/products/ft/ft_models.aspx?id=Delta) is mounted on top of the model and rigidly connected to the support by stiffeners. The FT measures all six components of force and torque from all three axes (x, y, and $\mathrm{z}$ ).

The origin is placed at the centre of the structure at SWL, as seen in Figure 5. All results have the origin as point of reference. Froude-based scaling of a conceptual prototype is applied. Table 3 shows the model specifications.

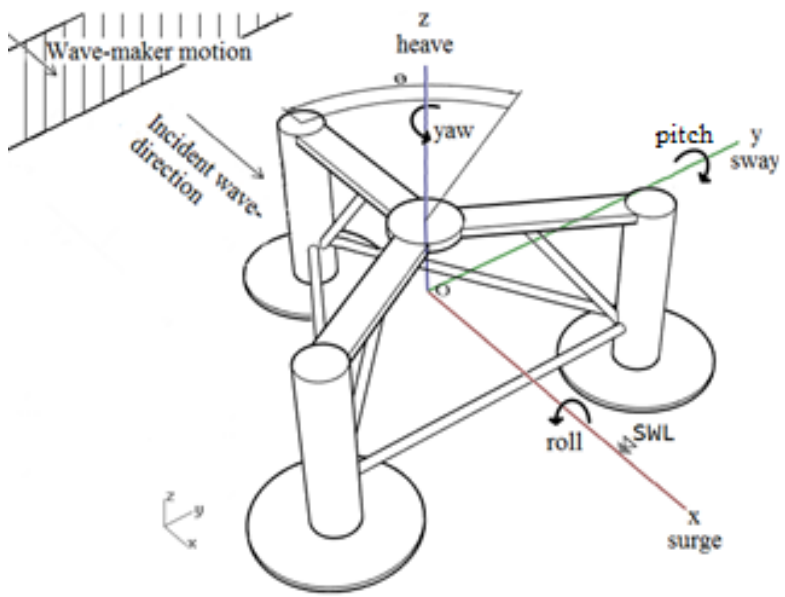

(a)

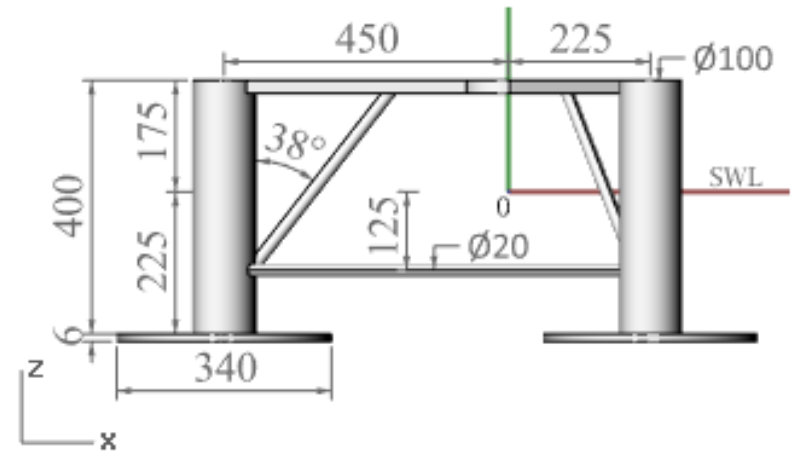

(b)

Figure 5. (a) Notation of DoFs according to [5]; (b) model dimensions [mm].

Table 3. Model specifications.

\begin{tabular}{ccc}
\hline Scale: $\sim \mathbf{1 / 8 0}$ & Model & Prototype \\
\hline Weight & $7.45 \mathrm{~kg}$ & $3469 \mathrm{ton}$ \\
$\mathrm{CoG}, \mathrm{z}_{(\mathrm{SWL}=0 \mathrm{~m})}$ & $-37 \mathrm{~mm}$ & $2.16 \mathrm{~m}$ \\
Draft & $225 \mathrm{~mm}$ & $18.00 \mathrm{~m}$ \\
\hline
\end{tabular}


Wave gauges are placed in the same distance from the wave maker as the model, but $2 \mathrm{~m}$ a side, see Figure 6.

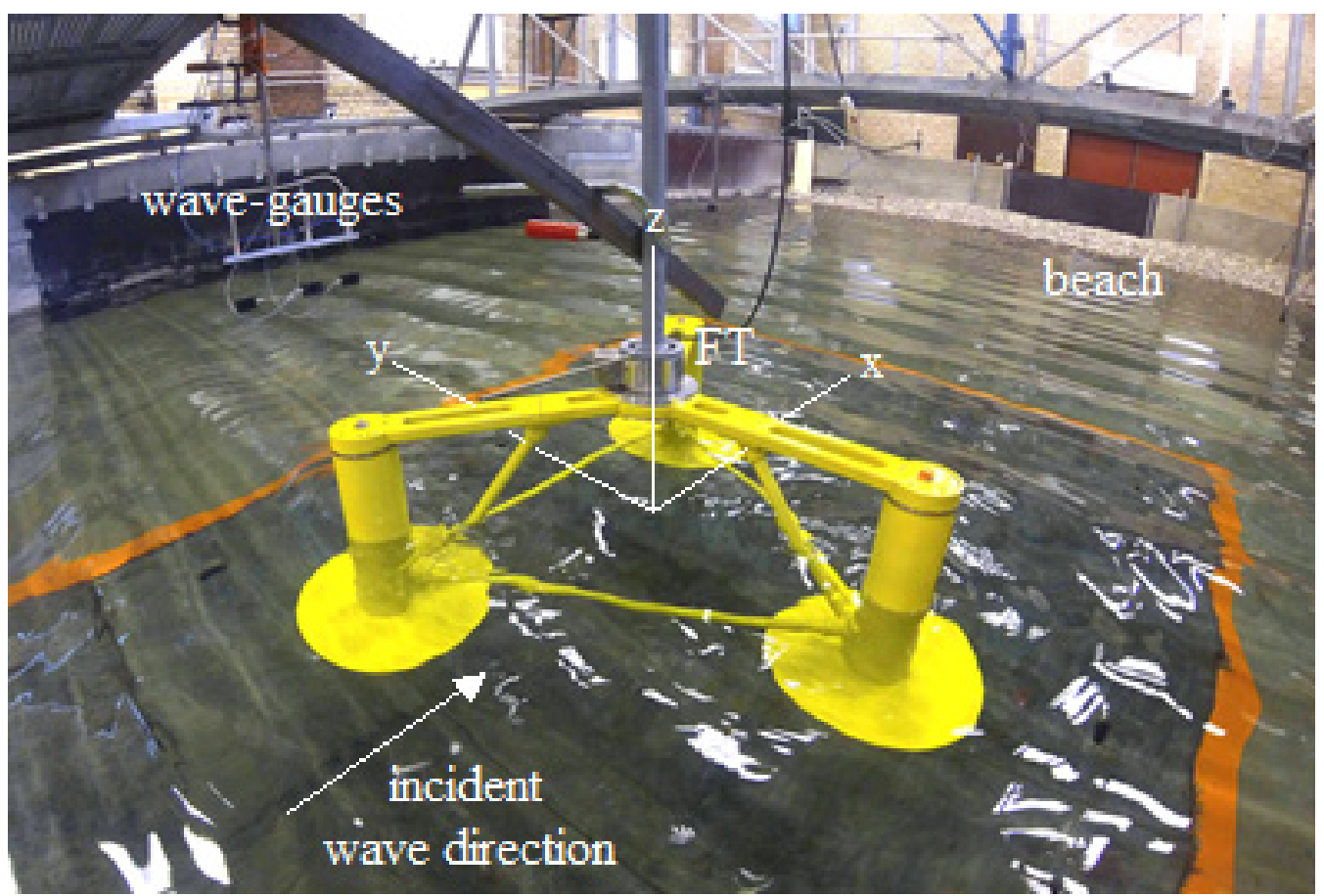

Figure 6. Picture of the model in the wave basin with illustration of the $x-, y$ - and z-axis.

\section{Results}

For data processing, an FFT analysis of the measuring signal is applied in the determination of T, $\mathrm{H}$ and force amplitude in surge $\left(F_{x}\right)$. The results are presented in graphs showing the parameters $\theta, f, H$ and modification of structure. All plots are normalized with respect to density ( $\rho$ ), gravity (g), wave height $(\mathrm{H})$, column radius (a) and water depth $(\mathrm{d})$.

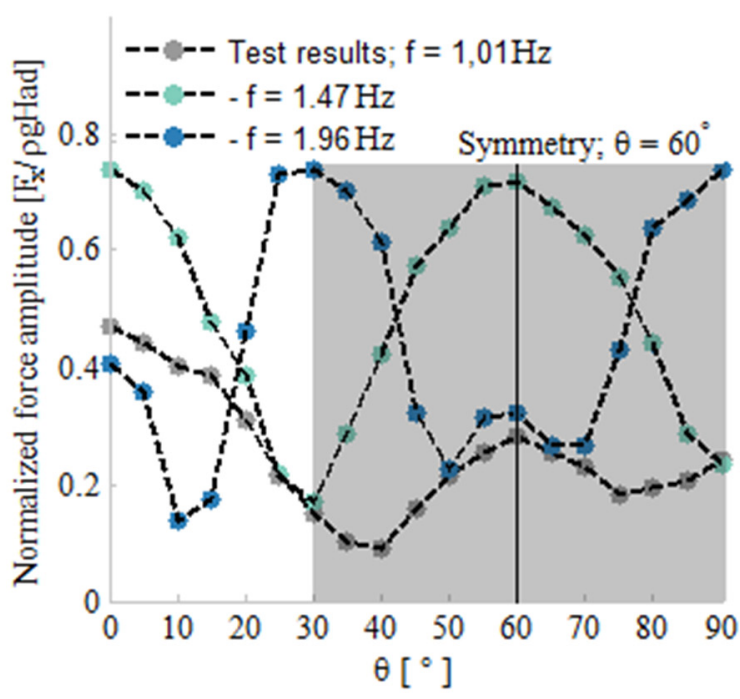

(a)

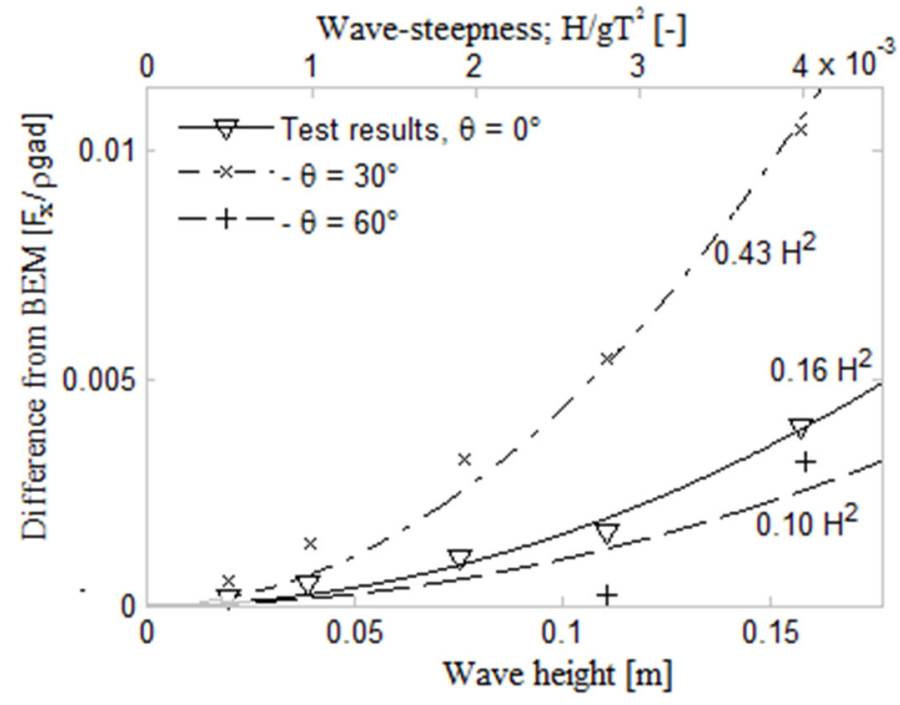

(b)

Figure 7. (a) Variation of $F_{x}$ against $\theta$ on model with heave-plates (b) $F_{x}$, difference between test results with heave-plates and Nemoh by increasing $H$. 
As presented in Section 2, the scatter plot and test results symbols are linked to the variation of $\mathrm{f}$ (interval of $0.1 \mathrm{~Hz}$ ) and, thereby, the dominant force regime, see Figure 2b. Figure $7 \mathrm{a}$ shows the normalized $\mathrm{F}_{\mathrm{x}}$ by the influence of $\theta$ with an interval of $5^{\circ}$ and constant $\mathrm{f}$. Peaks are observed around $0^{\circ}$, $30^{\circ}, 60^{\circ}$ and $90^{\circ}$. The tendency of the test results substantiate the expected axis of symmetry at $60^{\circ}$, with manual configuration of $\theta$ taken into account. Figure $7 \mathrm{~b}$ shows that by increasing $\mathrm{H}$ the test results diverge exponentially to Nemoh, which is due to violation of assumptions in potential theory. The influence of incident wave direction is considered to be caused by uncertainties in measurements, where non-symmetry affects the FT by moments around z-axis.

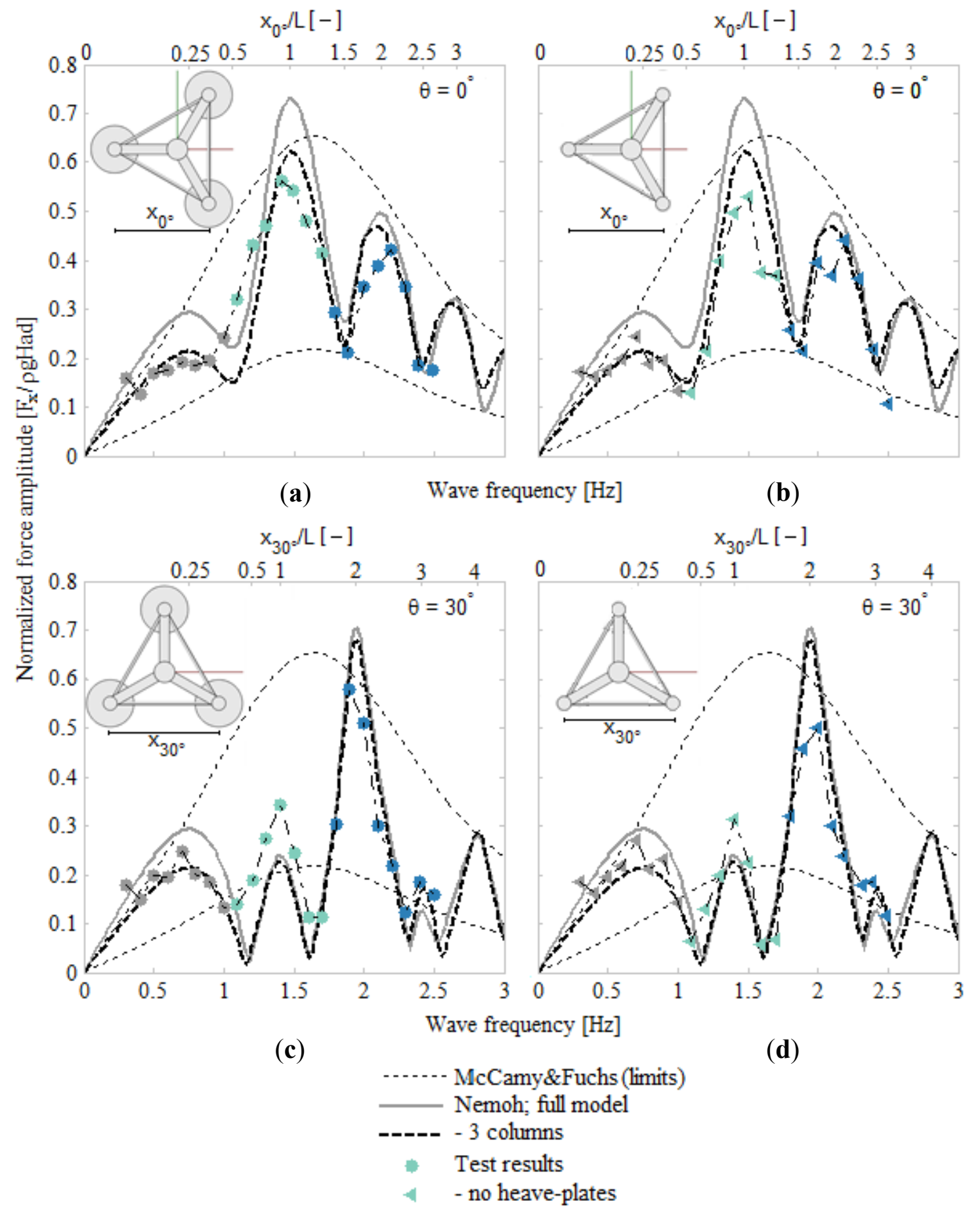

Figure 8. Fx force exerted at (a) $\theta=0^{\circ}$ (b) $\theta=0^{\circ}$, - no heave-plates $(\mathbf{c}) \theta=30^{\circ}$ (d) $\theta=30^{\circ}$, - no heave-plates. 
In Figures 8 and $9 \mathrm{a}, \mathrm{F}_{\mathrm{x}}$ is plotted by influence of $\theta$, $\mathrm{f}$ and $x_{\theta} / \mathrm{L}$, where $x_{\theta}$ is the maximum distance (in the incident wave direction) between the columns. As expected, the local maxima and minima can be expressed by the ratio $x_{\theta} / \mathrm{L}$, where maxima occurs at integers. The Nemoh-computed full model results diverge more to the test results at the peaks than with computation of primary structural parts only. The test results without heave-plates follow Nemoh better at local minima.

In Figure $9 b$, the normalized moment amplitude $\left(\mathrm{M}_{\mathrm{z}}\right)$ of excitation moment in yaw is plotted by influence of $\theta$, f and $x_{30^{\circ}} / \mathrm{L}$. The Nemoh computation of $\theta=0^{\circ}$ and $60^{\circ}$ have $\mathrm{M}_{\mathrm{z}}=0$ due to symmetry, thus the test results diverge with a light increasingly small amplitude at higher frequencies. The moment amplitude for $\theta=30^{\circ}$ follows the Nemoh computation for the full model.

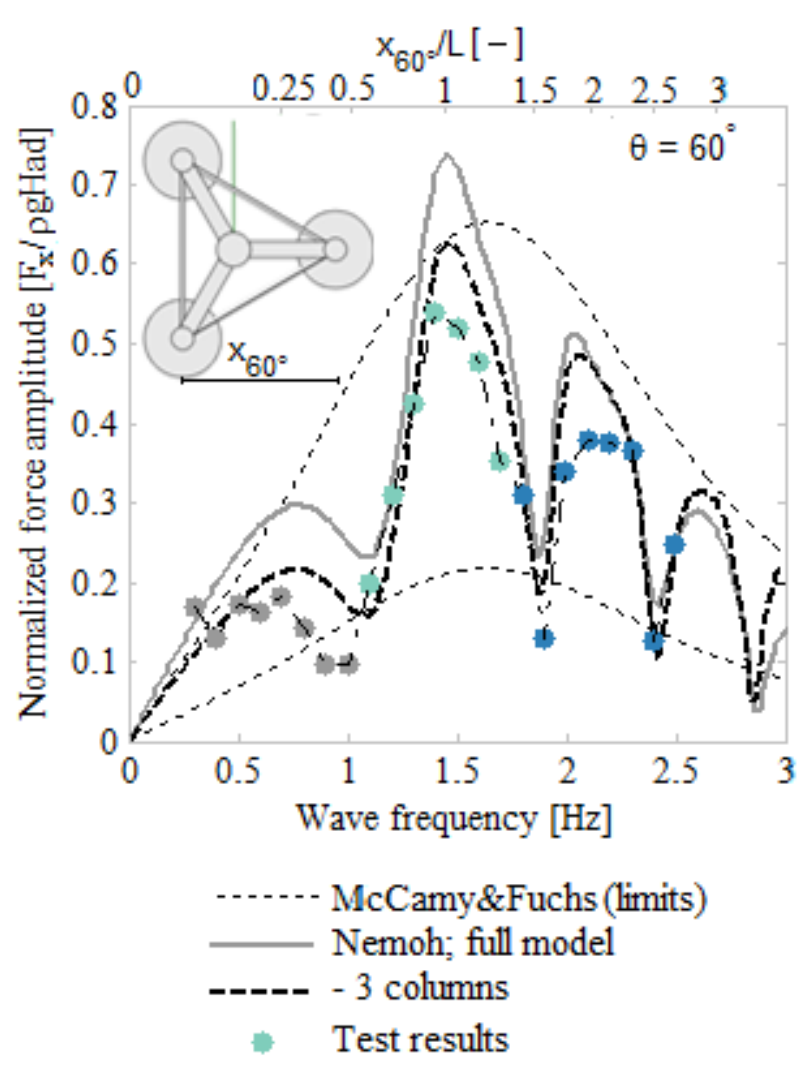

(a)

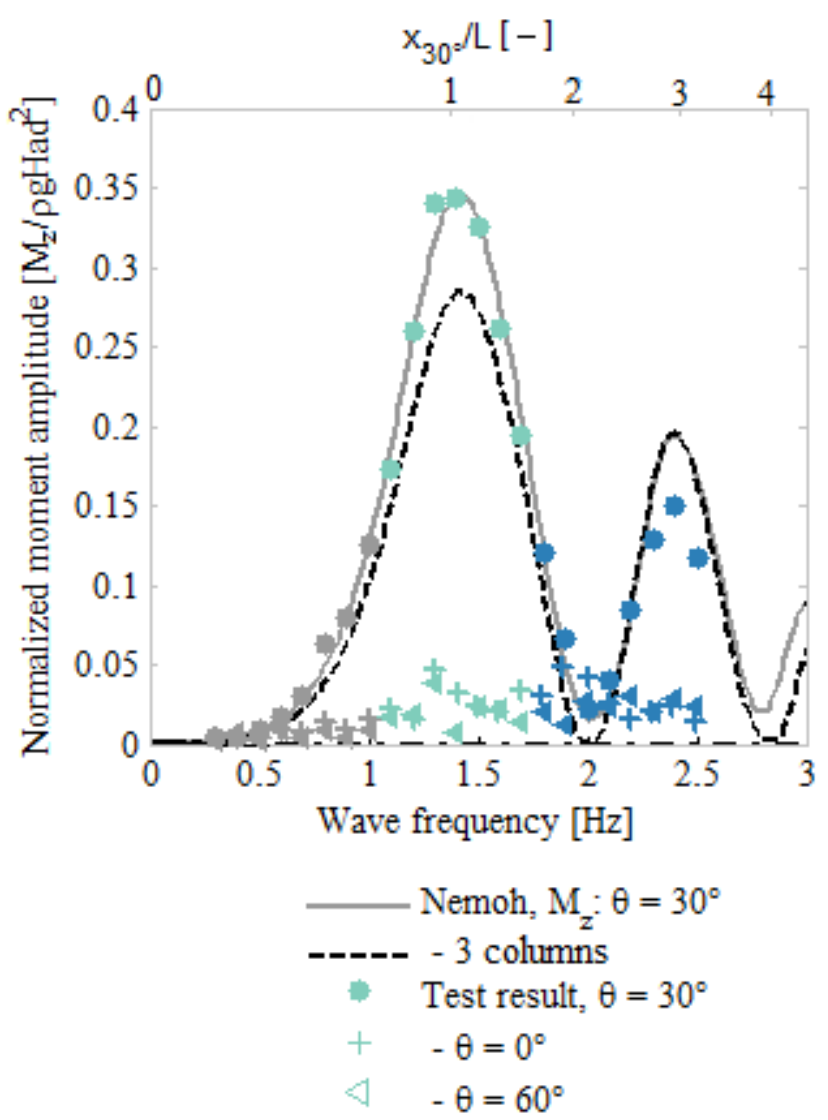

(b)

Figure 9. (a) $\mathrm{F}_{\mathrm{x}}$ force exerted, $\theta=60^{\circ}$ (b) moment exerted in yaw, $\mathrm{M}_{\mathrm{z}}$.

\section{Discussion}

Figures 8 and $9 \mathrm{a}$ are a comparison of the frequency dependent excitation force for three different directions. The force is almost similar for low frequencies, but with higher frequencies it depends on the incident wave direction. The excitation force for $0^{\circ}$ and $60^{\circ}$ is generally in the range of lower and upper limits by the McCamy and Fuchs solution whereas the interval for $30^{\circ}$ exceeds the lower limits due to a three-positioned placement of columns parallel to the incident wave direction. As seen in Figures 8 and 9a, the frequency for all local minima and maxima can be expressed by the geometric ratio $x_{\theta} / \mathrm{L}$, which leads to a simple determination of peak frequency and peak force regime. 
By determining the peak frequency and corresponding force regime, it is possible to solve excitation forces for peaks only and thereby decrease the number of equations $\left(n_{E Q}\right)$ significantly, see Equation (3):

$$
n_{E Q}=\left(n_{D o F s}+n_{\theta}\right) \cdot n_{f}
$$

where $n_{D o F s}, n_{\theta}$ and $n_{f}$ is number of: DoFs, incident wave direction and frequencies.

Table 4 shows the influence of $n_{f}$ and number of panels in computation time. The number of panels is observed to affect the computation time with a factor of $\sim 8$ times the ratio between meshes where $\mathrm{n}_{\mathrm{f}}$ affects it linearly.

Table 4. Duration of Nemoh computation for applied meshes and frequencies (where $n_{D o F s}=n_{\theta}=3$ ).

\begin{tabular}{cccc}
\hline Mesh & Frequencies & Time & Time [\%] \\
\hline Full model & All $\left(\mathrm{n}_{\mathrm{f}}=150\right)$ & $54.1 \mathrm{~h}$ & $100 \%$ \\
$(1590$ panels $)$ & Peak $\left(\mathrm{n}_{\mathrm{f}}=8\right)$ & $2.8 \mathrm{~h}$ & $5.3 \%$ \\
3 columns & All $\left(n_{f}=150\right)$ & $1.1 \mathrm{~h}$ & $2.1 \%$ \\
$(264$ panels $)$ & Peak $\left(n_{f}=8\right)$ & $3.6 \mathrm{~min}$ & $0.1 \%$ \\
\hline
\end{tabular}

* Nemoh v2.01 [13] on an Intel ${ }^{\circledR}$ Core $^{\mathrm{TM}} 3.00 \mathrm{GHz}$ CPU with $4.00 \mathrm{~GB}$ installed memory.

Figure 10 shows the difference between the Nemoh computed results of a mesh of the full model, a mesh of three columns only and the test results. As seen, the difference between computed results and test results is generally lower when only primary structural parts are computed. This is important because of the large difference in panels.

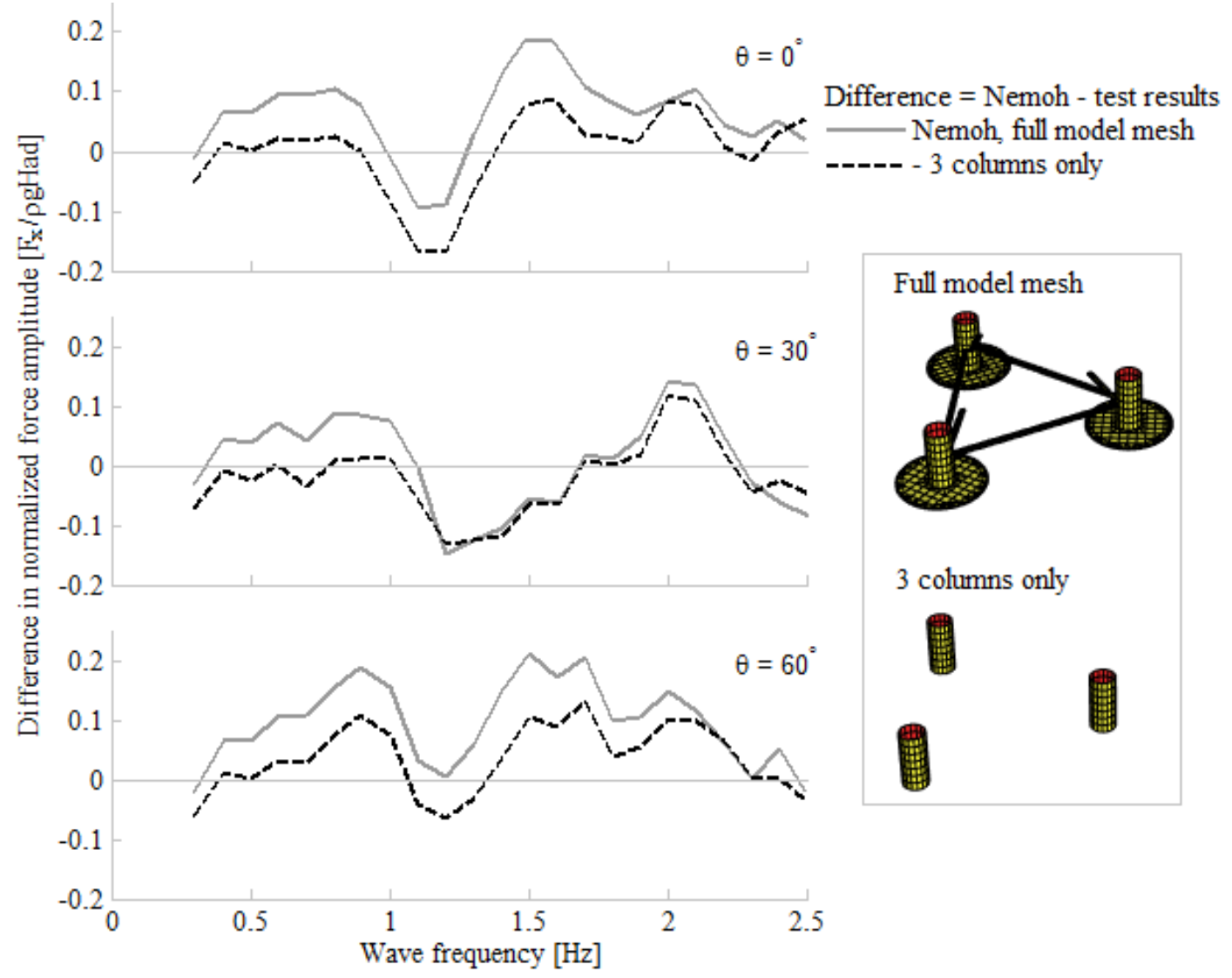

Figure 10. Difference between Nemoh-computed results and test results in normalized force amplitude. 
Figure 11 shows the simple computation of the three-columned mesh for peak frequencies only, which reduces the computation time to $0.1 \%$ of full model mesh for all frequencies (see Table 3 ). Despite the high reduction of computed panels and frequencies, the Nemoh computation is in good agreement with the test results. For the potential shadow effects in situation with in-line columns in incident wave direction $\left(\theta=30^{\circ}\right)$, the yaw excitation $\left(\mathrm{M}_{\mathrm{z}}\right)$ of the structure is considered, see Figure $9 \mathrm{~b}$. The results show that $\mathrm{M}_{\mathrm{z}}$ are close to zero for $0^{\circ}$ and $60^{\circ}$, and the deviation could be due to a small error in the angle of the wave direction. For $\theta=30^{\circ}$, a minimum close to zero is reached at $x_{30^{\circ}} / \mathrm{L}=2$ and, thereby, indicates the shadow effect as negligibly small. This corresponds to the assumption of a no interference effect for an in-line placed cylinder in an oscillating flow when $x_{30^{\circ}}>4 \mathrm{D}$ [17]. Since there is no indication of shadow effects, the theoretical solution by McCamy and Fuchs including consideration of re-positioning of columns is applicable and sufficient.

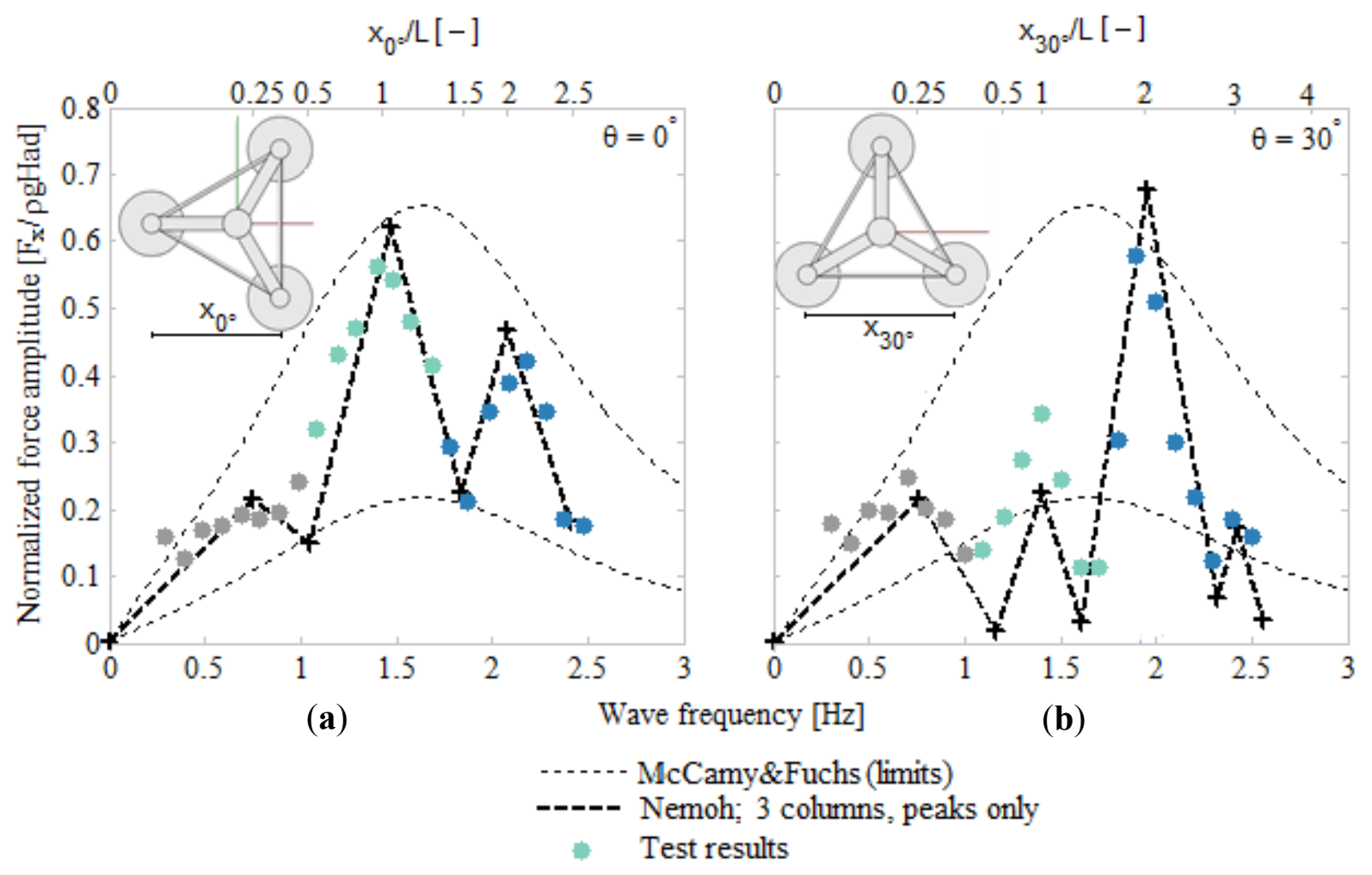

Figure 11. Nemoh computation of 3-columned model mesh for local peak frequencies at incident wave direction of (a) $\theta=0^{\circ}$ (b) $\theta=30^{\circ}$.

When increasing $\mathrm{H}$, the excitation force moves towards the drag regime, cf. Figure $2 \mathrm{~b}$. Because the calculation is based on linear diffraction theory, no viscous effects are taken into account and an increasing deviation in respect to the wave height is expected. Figure $7 \mathrm{~b}$ shows the normalized test result for a varying wave steepness at $\mathrm{f}=0.5 \mathrm{~Hz}$ in comparison with Nemoh. The test result deviates from Nemoh-computed results, which is considered to be primarily due to the increasing significance of the viscous effects and deviation from the linear wave elevation.

\section{Conclusions}

Because of the complex geometry, the determination of the hydrodynamic excitation force is doubtful due to the limitation of commonly used simplified methods. This paper shows good agreement between 
the test results of the applied model and the calculated results obtained by applying diffraction theory in the open source BEM code, Nemoh. Furthermore, the agreement between the calculated results and the test results gets stronger when applying a simplified panel mesh consisting of primary structural parts only. The peaks are especially estimated significantly better. The average difference between Nemoh computations and test results are around $40 \%$ higher for full model mesh than for model mesh of primary structures only which have $\sim 6$ times fewer mesh panels. By simplifying the model mesh into primary structures, more realistic solutions are thereby achieved. This can be caused by mitigating effects due to non-achievement of ideal situations of full wave excitation force on secondary and tertiary structural parts (heave-plates and braces).

The peak frequencies can be predicted by the ratio of the distance between the columns (in the incident wave direction) and the wavelength, which is why the necessary frequencies for computation, and as such the computation time, can be reduced significantly. By computing peak frequencies and mesh of primary structures only, the computation time is reduced to $0.1 \%$ of full model mesh for all frequencies. The results by reduced computation demonstrate a significantly more effective method which shows good agreement with the test results.

For low frequencies, the result with heave-plates diverge more from the ideal situation solved by potential theory than the results without heave-plates. This is due to the disturbance of the particle motion and the presence of skin friction over the heave-plates. By considering the excitation moment in yaw, the shadow effects of columns in surge are observed as negligibly small. This supports the assumption of a no interference effect for an in-line placed cylinder in an oscillating flow when the distance between columns (in incident wave direction) is higher than four times the column diameter.

\section{Acknowledgments}

The authors acknowledge the benevolence from Aalborg University, Department of Hydraulic and Coastal Engineering.

\section{Author Contributions}

MTA drafted and planned the work; MTA, DH and JL conceived and designed the experiments; DH and JL performed experiments, analysed data and drafted the paper under supervision of MTA; MTA, DH and JL finalized the paper.

\section{Conflicts of Interest}

The authors declare no conflict of interest.

\section{References}

1. International Renewable Energy Agency (IRENA). Renewable Eenergy Technologies: Cost Analasis Series, Volume 1: Wind Power; IRENA: Bonn, Germany, 2012.

2. European Wind Energy Association (EWEA). Deep Water; The Next Step for Offshore Wind Energy; EWEA: Brussels, Belgium, 2013. 
3. Jonkman, J.M. Dynamics of offshore floating wind turbines - model development and verification. Wind Energy 2009, 12, 459-492.

4. Hirdaris, S.; Bai, W.; Dessi, D.; Ergin, A.; Gu, X.; Hermundstad, O.A.; Huijsmans, R.; Iijima, K.; Nielsen, U.D.; Parunov, J.; et al. Loads for use in the design of ships and offshore structures. Ocean Eng. 2014, 78, 131-174.

5. Det Norske Veritas (DNV). DNV-RP-C205; Environmental Conditions and Environmental Loads; DNV: Høvik, Norway, 2010.

6. Sarpkaya, T. Wave Forces on Offshore Structures; Cambridge University Press: NewYork, NY, USA, 2010.

7. Morison, J.R.; O’Brien, M.P.; Johnson, J.W.; Schaaf, S.A. The force exerted by surface waves on piles. Petroleum Transactions. Am. Inst. Mining Eng. 1950, 189, 149-157.

8. NREL. Summary of Conclusions and Recommendations Drawn from the DeepCWind Scaled Floating Offshore Wind System Test Campaign. In Proceedings of the ASME 2013: 32nd International Conference on Ocean, Offshore and Arctic Engineering, Nantes, France, 9-14 June 2013.

9. Zhang, R.; Tang, Y.; Hu, J.; Ruan, S.; Chen, C. Dynamic response in frequency and timedomains of a floating foundation for offshore wind turbines. Ocean Eng. 2013, 60, 115-123.

10. Huijs, F.; Savenije, F.; de Bruijn, R. Concept design verification of a semi-submersible floating wind turbine using coupled simulations. Energy Procedia 2014, 53, 2-12.

11. Gao, Y.; Li, C.; Cheng, X. Research on foundation response of a tri-floater offshore wind. In IOP Conference Series: Materials Science and Engineering; IOP Publishing: Beijing, China, 2013; Volume 52.

12. Fernando, V.P. Semi-Submersible Topside Conceptual Design; Transition Piece. Master Thesis, Aalborg University, Esbjerg, Denmark, 2014.

13. LHEEA. Laboratoire de recherche en Hydrodynamique, Énergétique et Environnement Atmosphérique. Availale online: http://lheea.ec-nantes.fr/doku.php/en/start (accessed on 18 August 2014).

14. Massie, W.W.; Journée, J.M. Offshore Hydromechanics; Delft University of Technology: Delft, The Netherlands, 2001.

15. MacCamy, R.C.; Fuchs, R.A. Wave Forces on Piles: A Diffraction Theory; Technical Memorandum No. 69; Beach Erosion Board, U.S. Army Corps of Engineers, 1954.

16. AAU. Software for Wave Laboratories. Availale online: http:/www.hydrosoft.civil.aau.dk/awasys/ (accessed on 18 August 2014).

17. Frigaard, P.B.; Burcharth, H.F. Wave Loads on Cylinders; University of Aalborg: Esbjerg, Denmark, 1989.

(C) 2015 by the authors; licensee MDPI, Basel, Switzerland. This article is an open access article distributed under the terms and conditions of the Creative Commons Attribution license (http://creativecommons.org/licenses/by/4.0/). 